\title{
ARTICLE
}

Molecular Diagnostics

\section{Tumour-free distance: a novel prognostic marker in patients with early-stage cervical cancer treated by primary surgery}

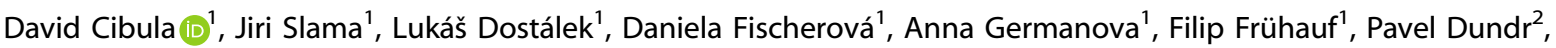 \\ Kristyna Nemejcova ${ }^{2}$, Jiri Jarkovsky ${ }^{3}$, Silvie Sebestova ${ }^{4}$, Andrea Burgetová ${ }^{5}$, Martina Borčinová ${ }^{1}$ and Roman Kocián ${ }^{1}$
}

BACKGROUND: Models predicting recurrence risk (RR) of cervical cancer are used to tailor adjuvant treatment after radical surgery. The goal of our study was to compare available prognostic factors and to develop a prognostic model that would be easy to standardise and use in routine clinical practice.

METHODS: All consecutive patients with early-stage cervical cancer treated by primary surgery in a single referral centre (01/ 2007-12/2016) were eligible if assessed by standardised protocols for pre-operative imaging and pathology. Fifteen prognostic markers were evaluated in 379 patients, out of which 320 lymph node (LN)-negative.

RESULTS: The best predictive model for the whole cohort entailed a combination of tumour-free distance (TFD) $\leq 3.5 \mathrm{~mm}$ and LN positivity, which separated two subgroups with a substantially distinct RR $36 \%$ and $6.5 \%$, respectively. In LN-negative patients, a combination of TFD $\leq 3.5 \mathrm{~mm}$ and adenosquamous tumour type separated a group of nine patients with RR $33 \%$ from the rest of the group with $6 \%$ RR.

CONCLUSIONS: A newly identified prognostic marker, TFD, surpassed all traditional tumour-related markers in the RR assessment. Predictive models combining TFD, which can be easily accessed on pre-operative imaging, with LN status or tumour type can be used in daily practice and can help to identify patients with the highest RR.

British Journal of Cancer (2021) 124:1121-1129; https://doi.org/10.1038/s41416-020-01204-w

\section{BACKGROUND}

Prognostic markers in early-stage cervical cancer are used to tailor the type of surgery, including lymph node (LN) staging, type of parametrectomy and, most importantly, the administration of adjuvant treatment. ${ }^{1,2}$ The multiple studies that have analysed prognostic markers in these patients in the last two decades differed substantially in numerous aspects, such as cohort size, source of the data, duration of the study interval, design, selection of evaluated markers and method of statistical analysis. ${ }^{3-19}$ The main limitation in the majority of studies was an insufficient standardisation of both clinical management during the study period, and, even more importantly, the method of assessment of individual markers. It is not surprising that the outcomes of these studies were inconsistent. Only a few prognostic markers were identified unanimously, including tumour size, stage of disease and LN involvement. Discrepant results were found for many other markers, such as age, lymphovascular space invasion (LVSI), depth of stromal invasion (DSI) or grade.

In an effort to avoid these limitations, we assessed the majority of traditional and a few rarely evaluated clinical and pathological variables in a large cohort of patients enrolled in a single institutional database. All cases were assessed by a standardised protocol for both pre-operative imaging and pathology. The goal of this study was to compare individual markers and identify those with the highest significance for the risk of recurrence and to develop a simple prognostic model, which would be easy to standardise and use in routine practice. An ideal model should differentiate subgroups of patients with the most profoundly different recurrence rate (RR).

\section{METHODS}

Patient selection

All consecutive patients with early-stage cervical cancer (stages $\mathrm{T} 1 \mathrm{a}-\mathrm{T} 2 \mathrm{~b})$ who were treated by primary surgery with curative intent in a single tertiary gynaecologic oncology centre from January 2007 to December 2016 were enrolled in the study. The main inclusion criteria consisted of: (a) histologically proven cervical cancer; (b) common tumour types: squamous cell carcinoma, adenocarcinoma or adenosquamous carcinoma; (c) stage T1a-T2b; (d) LNs not enlarged or suspicious on preoperative imaging; (e) primary surgical treatment with curative intent. Patients in whom radical hysterectomy or fertility-sparing procedure was abandoned intraoperatively and in whom pre-

\footnotetext{
${ }^{1}$ Gynecologic Oncology Center, Department of Obstetrics and Gynecology, First Faculty of Medicine, Charles University in Prague and General University Hospital in Prague, Apolinarska 18, Prague 2 12800, Czech Republic; ${ }^{2}$ Department of Pathology, First Faculty of Medicine, Charles University in Prague and General University Hospital in Prague, Studnickova 2 , Prague 2 12800, Czech Republic; ${ }^{3}$ Institute of Biostatistics and Analyses, Faculty of Medicine, Masaryk University, Kamenice 126/3 , Brno 62500, Czech Republic; ${ }^{4}$ Institute of Health Information and Statistics of the Czech Republic, Palackeho Namesti 4 P.O. Box 60, Prague 2 12801, Czech Republic and ${ }^{5}$ Department of Radiology, First Faculty of Medicine, Charles University in Prague and General University Hospital in Prague, U Nemocnice 499/2, Prague 2 12808, Czech Republic Correspondence: David Cibula (dc@davidcibula.cz)
} 


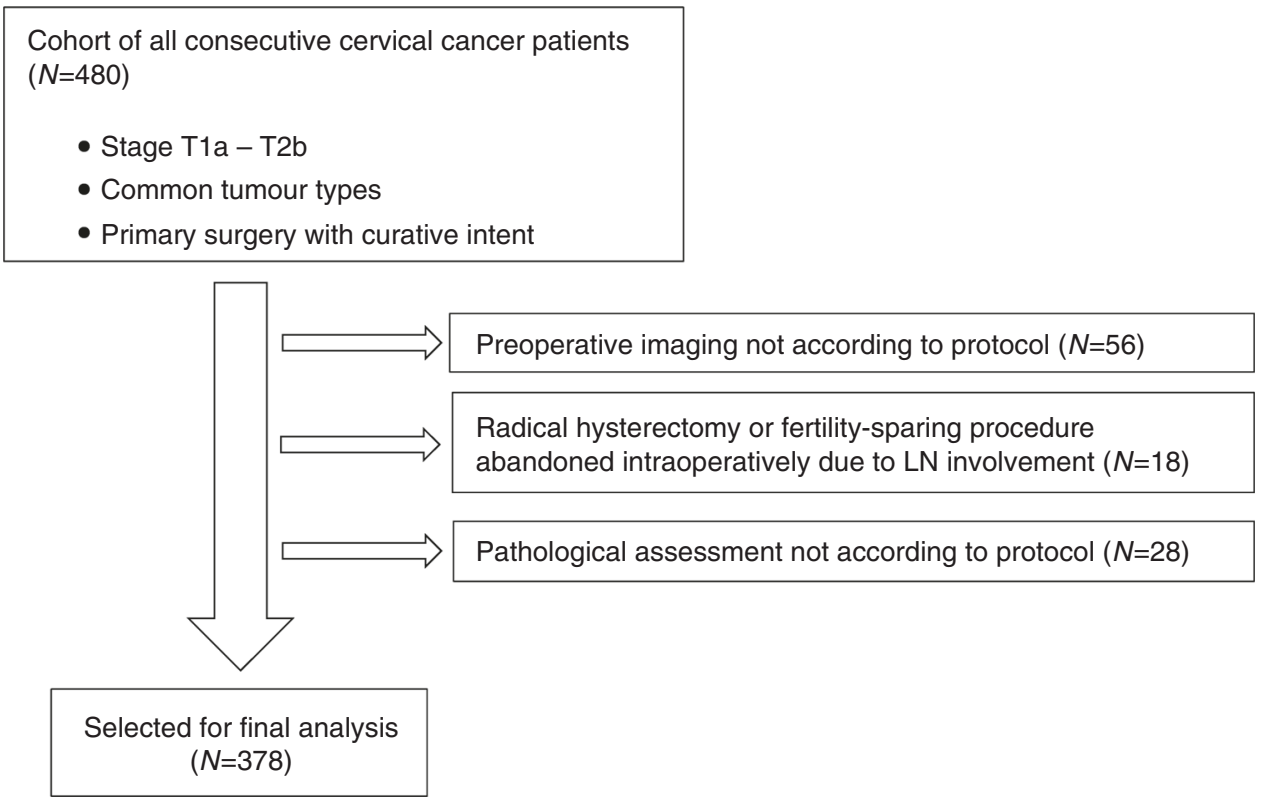

Fig. 1 Study flow chart.

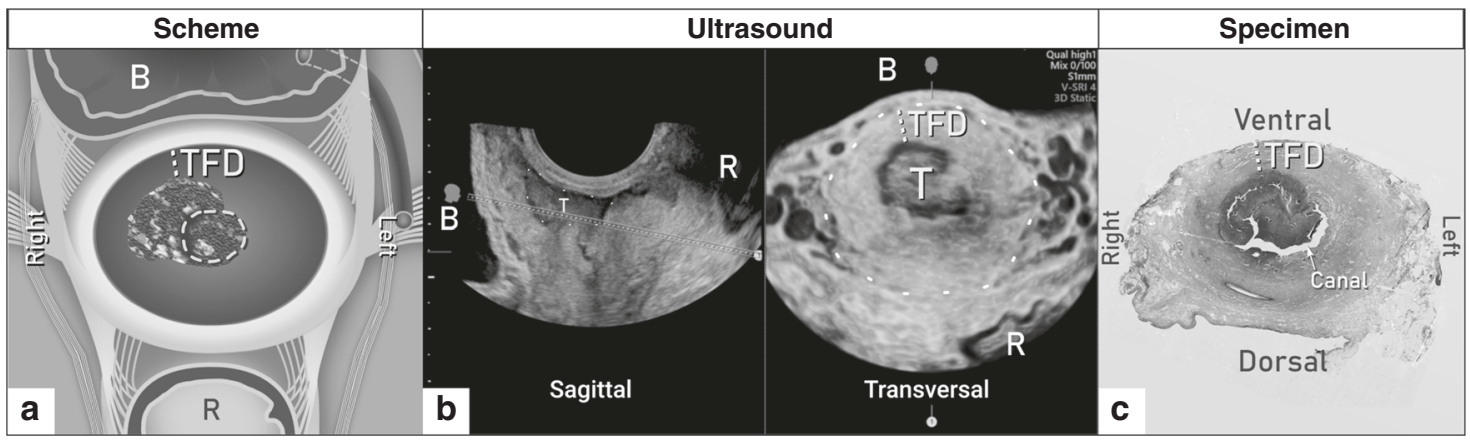

Fig. 2 Tumour-free distance (TFD) assessment. a Scheme of TFD measurement as the minimal lateral distance of uninvolved stroma between the tumour and pericervical ring; $\mathbf{b}$ TFD measurement by ultrasound in a transversal plane; $\mathbf{c}$ TFD measurement on pathological specimen.

operative imaging or pathological assessment was not performed according to standardised protocols were excluded (Fig. 1).

The study protocol was approved by the Institutional Review Board on 10/2017 under the registration number 1587/17 S-IV. Due to the retrospective nature of the study, a need for informed consent was waived.

Patient management

Surgical management has not been substantially changed within the time span of the study. Open surgery was the prevailing approach for radical hysterectomy or radical trachelectomy. The first step of the management was LN intraoperative assessment. SLN biopsy was performed selectively in the early period, and, it has become a standard part of the surgical management in our institution since 2010. If positive pelvic LN/SLN was detected during the surgery, cervical/uterine procedure was abandoned, and the patient was referred for primary chemoradiation (these cases were excluded from this analysis). In the majority of the patients, SLN biopsy was followed by a systematic pelvic lymphadenectomy (PLND), except for stage T1a/LVSI negative or in patients enrolled into a prospective SENTIX trial. The technique of systematic PLND remained unchanged and included seven anatomical regions in the pelvis, as previously described. ${ }^{20}$ The Querleu-Morrow classification was used for the description of the type of parametrectomy. ${ }^{21}$ The radicality of parametrectomy was tailored according to cervical prognostic risk factors. In stage T1a, a simple hysterectomy or conisation was performed; in higher stages, radical hysterectomy type C1 (nerve-sparing) was indicated in patients with smaller tumours, defined as a tumour-free distance $>0 \mathrm{~mm}$ (TFD, defined in "Pre-operative imaging" section) and the tumour size $\leq 3 \mathrm{~cm}$, while type $C 2$ was indicated in larger tumours and in those with no remaining TFD on either side of the cervix. Patients with LN involvement from the final pathology, patients with positive surgical margins or patients with parametrial invasion were referred to adjuvant treatment.

All cases were followed in the institution for at least 5 years after the treatment. Follow-up visits were scheduled every 3 months in the first 2 years, and in 6-12 months intervals thereafter, according to the presence of negative prognostic factors. One of the imaging tests (expert ultrasound or CT or PET/CT) was performed in all patients between 6 and 12 months interval after the surgery or after the adjuvant treatment has been finished. Further imaging tests were done only if clinically indicated, either in the presence of symptoms or any suspicious finding on physical examination. The diagnosis of recurrence was defined as (a) unequivocal finding on imaging; (b) suspicious recurrence on imaging either confirmed by biopsy or supported by other signs (disease progression on imaging or progression of symptoms) or 
death caused by disease or death of unknown cause. The outcome of patients was matched with the Czech National Database of Death Certificates, so mortality data and a cause of death were verified.

\section{Pre-operative imaging}

In the first period, 2000-2006, the combination of magnetic resonance imaging (MRI) and expert ultrasound (US) was routinely used. $^{22}$ Given better results of US in the local assessment, patients were examined only by US during the following period. ${ }^{22-24}$

US images were obtained with a GE Logic 9 and Voluson E8 ultrasound machine (GE Medical Systems, Milwaukee, WI, USA) equipped with an endoluminal microconvex linear array probe of 5-9 MHz frequency and a transabdominal convex linear array probe of $5 \mathrm{MHz}$ frequency. Patients were examined in the lithotomy position with an empty bladder. Transrectal placement of the probe was preferred to transvaginal approach, not only due to the lower risk of tumour bleeding but the evaluation of the distal part of the cervix is often less hampered by artefacts (acoustic shadows) and it allows for a closer distance between the probe and the tumour. ${ }^{25}$

All patients underwent a standardised US examination by an experienced examiner (level 3 according to the recommendations for the practice of the medical US of the European Federation of

Table 1. Summary of demographic and clinical parameters in the whole cohort and in lymph node (LN)-negative patients.

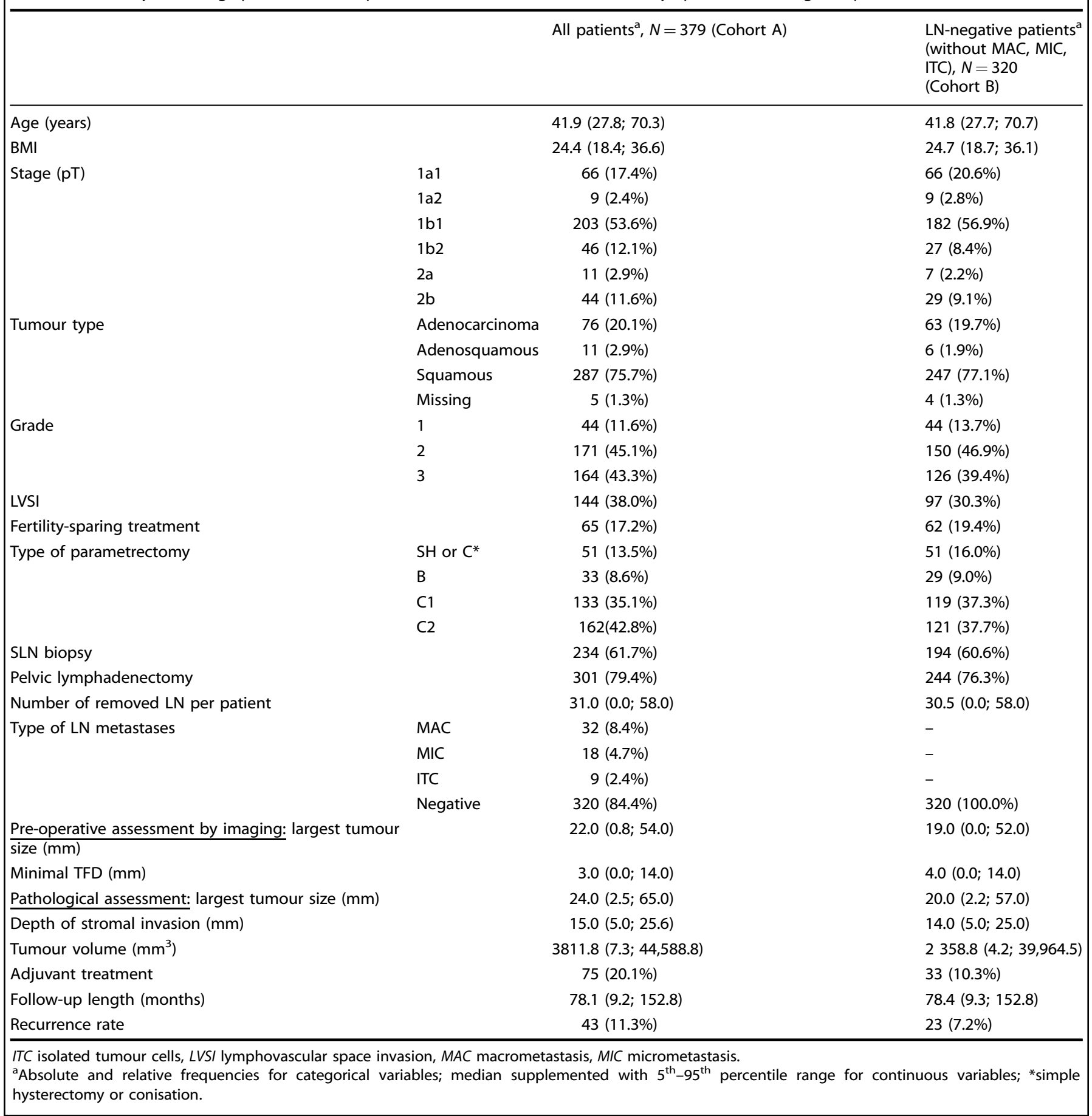


1124

Societies for Ultrasound in Medicine and Biology); ${ }^{26}$ the methodology was described in more detail elsewhere. ${ }^{27}$ Using the realtime 2D-US (greyscale and power Doppler), the examiner followed a standardised protocol and evaluated the presence of the tumour, the tumour size in three diameters, the distance between the cranial pole of the tumour and the internal cervical os, the integrity of the pericervical ring, the involvement of parametria and adjacent organs and the retroperitoneal LNs (Supplementary Table S1). TFD was measured on a transversal plane as a minimum distance of uninvolved stroma between the tumour and pericervical ring (dense hyperechogenic layer surrounding the cervix) (Fig. 2). If no residual tumour was found by imaging after conisation, TFD was calculated as half of the cervical transversal diameter. A standardised US protocol was completed in the webbased central database, data were locked after an examination, and the database did not allow any subsequent changes. When clinically indicated, or if any limitation of US was encountered, the appropriate complementary imaging method was completed and reviewed by an experienced radiologist dedicated to gynaecologic oncology.
Pathological assessment

For the frozen section, every SLN/ LN was bisected, and each half was examined. Each SLN was processed as a whole by cutting 2$\mathrm{mm}$-thick slices perpendicular to its long axis and analysed using ultrastaging protocol, as described previously. ${ }^{28}$ Non-SLNs were processed completely, in 2-mm-thick slices. LNs smaller than 3 $\mathrm{mm}$ were not sectioned.

Processing of the hysterectomy specimen consisted of macroscopic description, grossing, sampling and histological examination which were carried out in accordance with a standardised institutional protocol reflecting international and national guidelines. Each biopsy report was accompanied by a checklist summarising all relevant markers, such as tumour size in three diameters, tumour location, relationship to surrounding structures and presence of LVSI. A detailed description of the hysterectomy specimen processing can be found in Supplementary Table S2.

Prognostic markers

The method used for the assessment of individual markers is reported in the article as " $\mathrm{P}$ " for pathology or " $\mathrm{I}$ " for imaging.

Table 2. Univariate analysis of recurrence predictors.

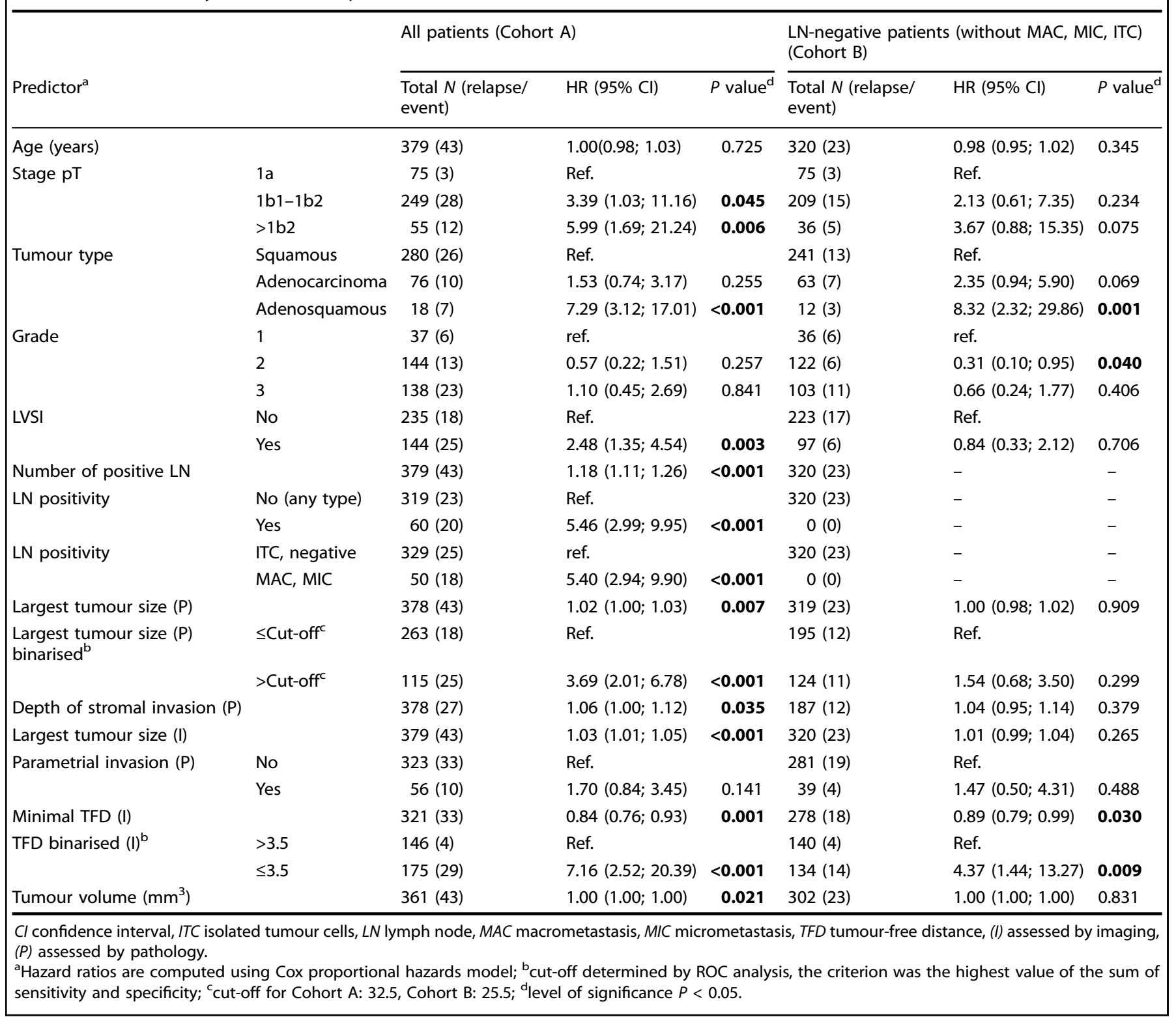


Tumour-free distance: a novel prognostic marker in patients with... D Cibula et al.

Together, 15 prognostic markers were evaluated, including age, 11 tumour-related and three LN status-related ones (LN positivity, number of positive LNs; type of metastasis in LN). Amongst tumour-related factors, seven were related to tumour size assessment: stage $(P)$, largest tumour size $(P$ and $I)$, tumour volume calculated by the formula for ellipsoid from pathological measurement $(P)$, largest tumour size binarised $(P)$, depth of stromal invasion (DSI) (P), minimal TFD (I) defined as the minimal uninvolved stroma between the tumour and pericervical ring (dense hyperechogenic layer on ultrasound while hypointense layer on MRI) on either side of the cervix, minimal TFD binarised (I); and four markers related to pathology or local disease spread: LVSI, tumour type, grade, parametrial invasion (P). Gynaecologic Oncology Group score (GOG score) was calculated according to the GOG criteria combining LVSI status, DSI and tumour size. ${ }^{3}$

Statistical analysis

Three groups of patients were analysed separately: (a) all patients enrolled in the study; (b) patients without LN involvement (after excluding cases with micrometastases, micrometastases, and isolated tumour cells); (c) patients who did not receive adjuvant treatment.

Standard descriptive statistics were applied in the analysis; absolute and relative frequencies for categorical variables and median supplemented with the 5th-95th percentile range for continuous variables. The influence of patients' characteristics on their recurrence risk was analysed using univariate and multivariate Cox proportional hazards models and described using hazard ratios (HRs) and their 95\% confidence intervals. The multivariate model was computed using a forward stepwise algorithm on a subset of predictors statistically significant in univariate analysis. Cut-off values for continuous variables were determined by ROC analysis; the criterion was the highest value of the sum of sensitivity and specificity. AUC from ROC analysis for multivariate models was adopted as a measure of their overall predictive power. Kaplan-Meier methodology was adopted for the visualisation of recurrence-free survival (RFS) data according to patient categories derived from variable combinations in multivariate models; the statistical significance of RFS curve differences among groups of patients was tested using the log-rank test. The analysis was computed using SPSS 25.0.0.1 (IBM Corporation 2018).

\section{RESULTS}

Group characteristics

Table 1 presents the characteristics of two cohorts, the whole group $(N=379)$ and the $L N$-negative group $(N=320)$ after all cases with any type of metastasis (macrometastases (MAC), micrometastases (MIC) and isolated tumour cells (ITC)) were excluded.

LN involvement was detected in 59 cases (16\%), from which $8.4 \%$ were MAC, $4.7 \%$ MIC and $2.4 \%$ ITC. The surgical approach was mostly open surgery. Only $22(7 \%)$ radical hysterectomies were performed by laparoscopy. Sentinel lymph node (SLN) biopsy was not performed in 145 cases (38\%): due to the failure of detecting $\operatorname{SLN}(N=30)$, early disease stages which did not require LN assessment (stage T1a1 or T1a2/ LVSI neg) $(N=59)$, cases prior to $2009(N=32)$ when SLN started to be performed routinely, and other reasons $(N=24)$. Pelvic lymph node dissection (PLND) was not performed in 78 cases (21\%): 69 of them were in stage T1a with either no LN staging or just SLN biopsy, and 9 patients after 2016 were enrolled into the prospective SENTIX trial, in which part of the management is SLN biopsy only, without PLND. In the whole cohort, 75 patients $(20 \%)$ were referred to adjuvant treatment for the following reasons: positive parametria (11), positive vaginal margins (2), positive LN (59), other reasons (3) (for details see Supplementary Table S3). The RR reached $11.3 \%$ in the

\begin{tabular}{|c|c|c|c|}
\hline Whole cohort & & $\mathrm{HR}(95 \% \mathrm{Cl})$ & $P$ value \\
\hline \multicolumn{4}{|l|}{ Model 1} \\
\hline \multicolumn{2}{|l|}{ Minimal TFD (I) } & $0.85(0.77 ; 0.94)$ & 0.002 \\
\hline \multicolumn{2}{|l|}{ Number of positive LN } & $1.14(1.05 ; 1.23)$ & 0.001 \\
\hline \multicolumn{4}{|l|}{ Model 2} \\
\hline TFD (I) binarised ${ }^{\mathrm{a}}$ & $\leq 3.5$ (ref. >3.5) & $4.58(1.52 ; 13.80)$ & 0.007 \\
\hline LN positivity & Yes (ref. no) & $3.79(1.83 ; 7.81)$ & $<0.001$ \\
\hline \multicolumn{4}{|l|}{ Model 3} \\
\hline TFD (I) binarised ${ }^{a}$ & $\leq 3.5$ (ref. >3.5) & $4.57(1.50 ; 13.97)$ & 0.008 \\
\hline LN positivity & Yes (ref. no) & $3.76(1.67 ; 8.50)$ & 0.001 \\
\hline LVSI & Yes (ref. no) & $1.01(0.45 ; 2.28)$ & 0.976 \\
\hline \multicolumn{4}{|l|}{ Model 4} \\
\hline TFD (I) binarised ${ }^{\mathrm{a}}$ & $\leq 3.5$ (ref. >3.5) & $3.48(0.71 ; 17.00)$ & 0.123 \\
\hline LN positivity & Yes (ref. no) & $5.02(2.26 ; 11.14)$ & $<0.001$ \\
\hline Stromal invasion $(\mathrm{P})$ & & $1.00(0.94 ; 1.07)$ & 0.989 \\
\hline \multicolumn{4}{|l|}{ Model 5} \\
\hline TFD (I) binarised ${ }^{\mathrm{a}}$ & $\leq 3.5$ (ref. >3.5) & $4.13(1.16 ; 14.69)$ & 0.029 \\
\hline LN positivity & Yes (ref. no) & $3.8(1.82 ; 7.92)$ & $<0.001$ \\
\hline \multirow[t]{2}{*}{ Tumour type } & Adeno (ref. squamo) & $1.39(0.26 ; 7.49)$ & 0.700 \\
\hline & $\begin{array}{l}\text { Adenosquamo (ref. } \\
\text { squamo) }\end{array}$ & $1.24(0.2 ; 7.78)$ & 0.816 \\
\hline \multicolumn{4}{|l|}{ Model 6} \\
\hline $\begin{array}{l}\text { Largest tumour size }(P) \\
\text { binarised }^{\mathrm{a}}\end{array}$ & $>32.5$ (ref. $\leq 32.5$ ) & $2.29(1.16 ; 4.49)$ & 0.016 \\
\hline LN positivity & Yes (ref. no) & $3.78(1.94 ; 7.36)$ & $<0.001$ \\
\hline \multicolumn{4}{|c|}{$\begin{array}{l}\text { adeno adenocarcinoma, CI confidence interval, } L N \text { lymph node, LVSI } \\
\text { lymphovascular space invasion, squamo squamous cell cancer, TFD tumour- } \\
\text { free distance, (I) assessed by imaging, (P) assessed by pathology. } \\
{ }^{a} \text { Cut-off determined by ROC analysis, the criterion was the highest value of } \\
\text { the sum of sensitivity and specificity. }{ }^{b} \text { level of significance } P<0.05 \text {. }\end{array}$} \\
\hline
\end{tabular}

whole group and $7.2 \%$ in the LN-negative cohort with a median follow-up of 78 months in both groups.

Univariate and multivariate analyses of prognostic markers Fifteen markers were evaluated in univariate analysis for predicting RR (Table 2). The highest hazard ratio (HR) was found for adenosquamous tumour type (HR $7.29(3.12 ; 17.01)$ ), stage $\geq 1 \mathrm{~b} 2$ (HR 5.99 (1.69; 21.24)), LN involvement (HR 5.46 (2.99; 9.95)), tumour size $\geq 32 \mathrm{~mm}$ (HR $3.69(2.01 ; 6.78)$ ), and tumour-free distance (TFD) $\leq 3.5 \mathrm{~mm}$ (HR $7.16(2.52 ; 20.39)$ ). TFD cut-off was determined by ROC analysis, the criterion was the highest value of the sum of sensitivity and specificity. All markers related to the tumour size were significant (largest tumour size assessed by pathology $(P)$, tumour volume $(P)$, minimal TFD assessed by imaging (I), DSI (P) and tumour volume (P)). Only four markers were significant in the cohort of $L N$-negative patients (adenosquamous tumour type, grade 2, minimal TFD, TFD $\leq$ $3.5 \mathrm{~mm}$ ).

All significant markers related to RR in univariate analyses were included in multivariate analyses using the Cox proportional hazards model (Table 3). Comparison of models according to AUC values is shown in Fig. 3. There were no major differences between Models 1 and 5 (AUC between 0.751 and 0.768). Model 2 was selected as the simplest, composed of two factors only, both in binary format: LN positivity and TFD $\leq 3.5 \mathrm{~mm}$. Combining the model with additional tumour-related markers, such as LVSI, DSI or tumour type, did not substantially improve the reliability of the model. Replacing TFD with the largest tumour size $(>32.5 \mathrm{~mm})$ substantially decreased the model's reliability (AUC $=0.700$ ).

Figure 4 shows the Kaplan-Meier RFS curve for Model 2. RR in group one $(\mathrm{NO}+\mathrm{TFD} \geq 3.5 \mathrm{~mm})$ and two $(\mathrm{NO}+\mathrm{TFD}<3.5 \mathrm{~mm})$ was 


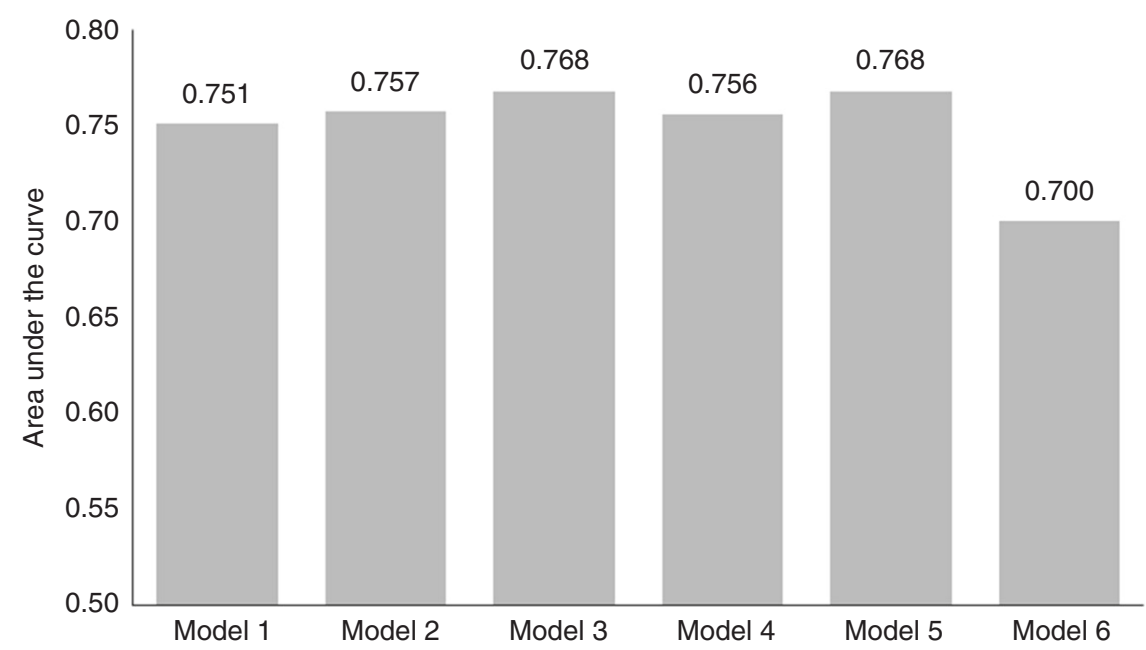

Fig. 3 Accuracy of prognostic models for recurrence risk in the whole cohort (ROC analysis). Model 1: Minimal tumour-free distance (TFD), number of positive lymph nodes (LN); Model 2: TFD binarised, LN positivity binarised; Model 3: TFD binarised, LN positivity binarised, lymphovascular space invasion; Model 4: TFD binarised, LN positivity binarised, depth of stromal invasion; Model 5: TFD binarised, LN positivity binarised, tumour type; Model 6: largest tumour size binarised, LN positivity binarised.

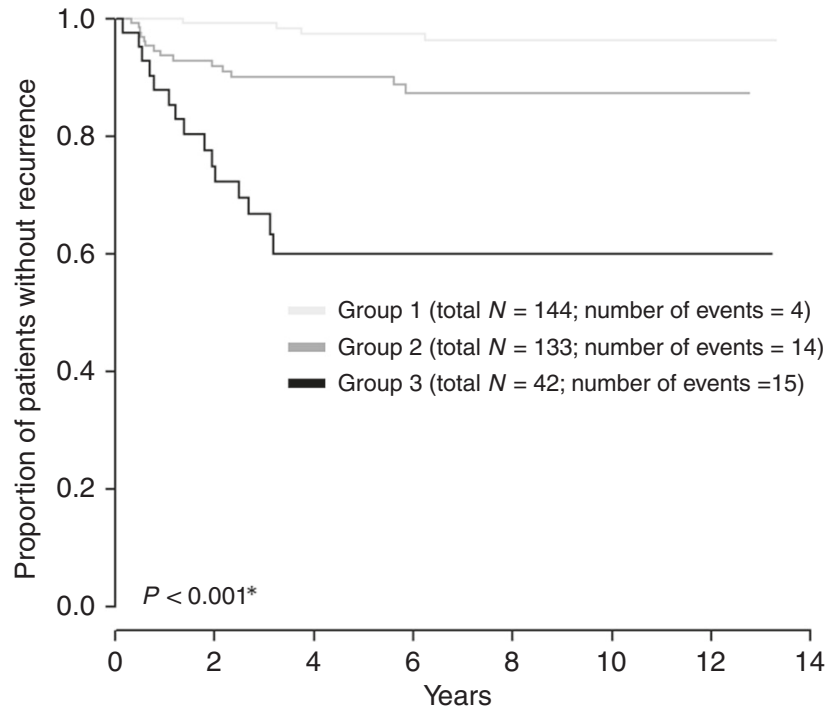

Fig. 4 Kaplan-Meier recurrence-free survival (RFS) curve for Model 2 in the whole cohort. Group description: Group 1: tumour-free distance (TFD) $>3.5^{* *}$, N0**; Group 2: TFD $\leq 3.5^{* *}$, $\mathrm{N} 0^{* *}$; Group 3: TFD $\leq 3.5^{* *}, \mathrm{~N} 1^{* *}$. Two patients without event are not included in the groups: minimal tu-pcf (US) $>3.5^{* *}$; a number of positive LN $>00^{* *}$. * Log-rank test. ${ }^{* *}$ Cut-off determined by ROC analysis, the criterion was the highest value of the sum of sensitivity and specificity.

$2.8 \%$ and $10.5 \%$, respectively, while in group three (TFD $<3.5 \mathrm{~mm}+$ N1) there were only 42 patients and the RR risk reached $36 \%$.

Models for LN-negative patients were composed of minimal TFD, tumour type, TFD $\leq 3.5 \mathrm{~mm}, \mathrm{LVSI}, \mathrm{DSI}$ and largest tumour size $>32.5 \mathrm{~mm}$ (Table 4 and Fig. 5). An additional model was calculated according to the GOG 92 study. ${ }^{3}$ There were substantial differences in accuracy between the models (Fig. 5). Model 2 was selected as the simplest and clinically most relevant. It included only two binary factors, TFD $\leq 3.5 \mathrm{~mm}$ and tumour type. The model became less accurate when TFD was replaced by the largest tumour size (Model 5, Table 4). The prognostic model constructed based on GOG intermediate-risk criteria, using traditional tumour-related risk factors, such as LVSI, tumour size, and DSI, were substantially less reliable. The Kaplan-Meier curve for Model 2 is shown in Fig. 6. It separated two groups, one of them with only nine cases and a RR of $33 \%$ (TFD $\leq 3.5$, adenosquamous tumour type), the other one with the majority of patients and a RR of only $6 \%$ (other combinations).

In addition, a separate analysis was conducted for the third group consisting solely of the patients who did not receive adjuvant treatment. In this cohort, only 18 recurrences and 9 deaths occurred. Univariate analysis proved the significance of the same prognostic factors as for the whole cohort. Two models were selected by multivariate analysis as the simplest and clinically most relevant, both consisting of only two parameters. The first model entailed TFD $\leq 3.5 \mathrm{~mm}$ and LN positivity (AUC $=0.746)$ and the second one consisted of TFD $\leq 3.5 \mathrm{~mm}$ and the number of positive $\mathrm{LNs}$ ( $\mathrm{AUC}=0.778$ ).

\section{DISCUSSION}

In our large cohort of patients assessed by standardised protocols for pre-operative imaging and pathological assessment, we evaluated 15 tumour-related or LN-related prognostic markers. Adenosquamous tumour type, LN involvement and TFD $\leq 3.5 \mathrm{~mm}$ were the most significant independent factors for the risk of recurrence in the whole cohort. By combining factors significant from univariate analysis, we created simple prognostic models, entailing only two factors, both in a binary format that defined a small subgroup of patients with a significantly higher RR. In the entire cohort, the combination of LN involvement and TFD $\leq 3.5$ defined a group of $13 \%$ of cases with a RR of $36 \%$, whereas, in the rest of the cohort, the RR reached only $6.5 \%$. In the cohort of LN-negative patients, the combination of adenosquamous tumour type and TFD $\leq 3.5$ defined a group of $3 \%$ of cases with a RR of $33 \%$ in comparison to a $6 \%$ RR in the rest of the cohort.

Dozens of studies have assessed prognostic factors in the past, which largely varied in size, disease stage and study interval length. ${ }^{3-19}$ The majority of these studies were retrospective analyses that did not define standards of care. It should be emphasised that the selection of patients, radicality of the surgery or criteria for adjuvant treatment are all aspects that can alter the oncological outcome and, therefore, also the significance of individual prognostic factors. Another potential bias is the frequently missing standardised methodology for the assessment 
Table 4. Multivariate analysis of prognostic markers in LN-negative patients.

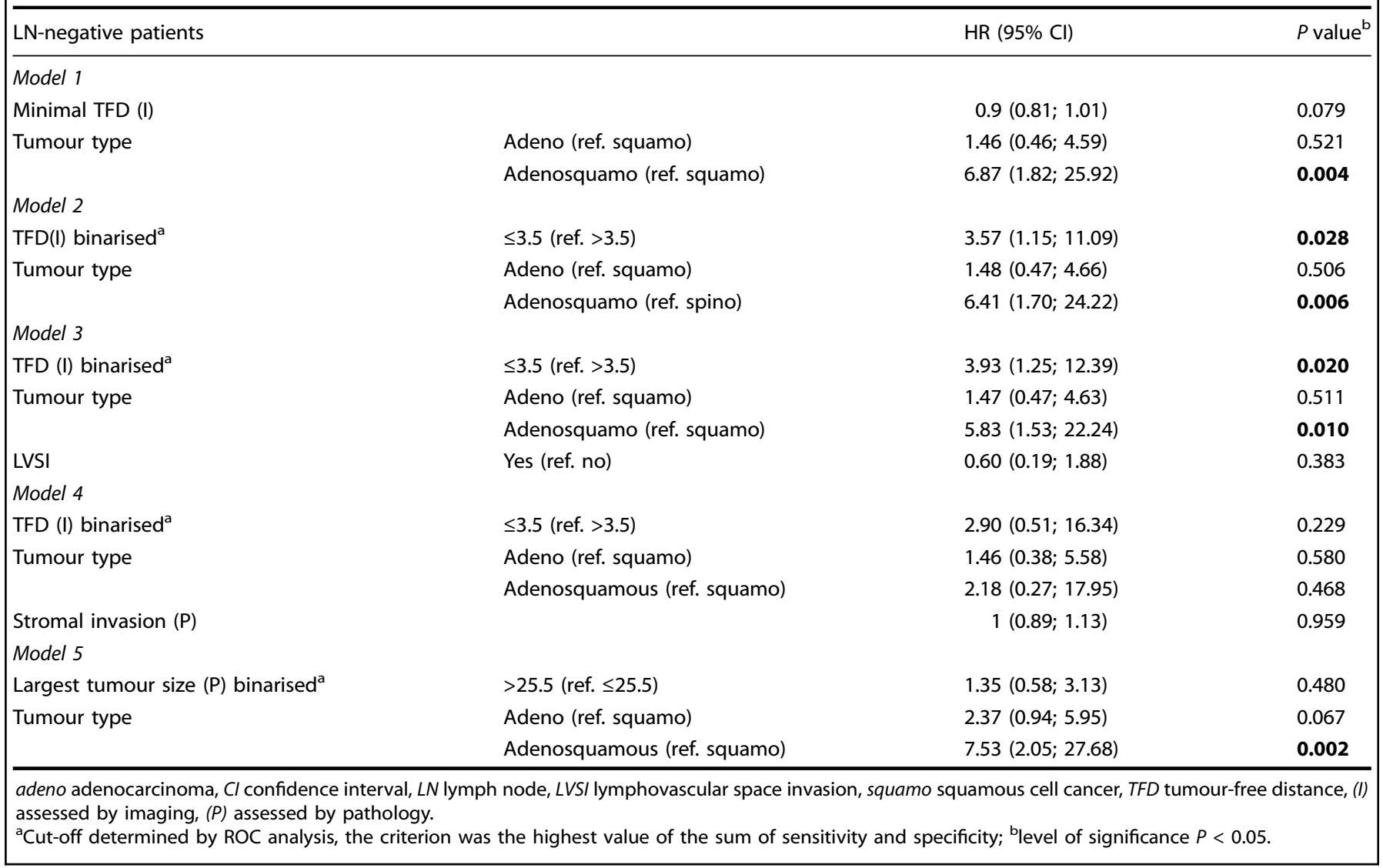

of individual prognostic factors. Without standardised methodology, the assessment of LN status or tumour-related markers (tumour size, stromal invasion, LVSI) become unreliable, especially in multicentre studies or if the data are retrieved from national databases.

In 2009, Biewenga et al. ${ }^{19}$ aimed to validate 12 published prognostic models ${ }^{3-14}$ in an independent population of 563 patients treated for early-stage cervical cancer. They found that the great majority of published models overestimated the risk of recurrence or death from disease in their validation group, especially in the higher-risk categories. Only two models were valid for the prediction of the recurrence-free or disease-specific survival in their patient population. Five-year recurrence-free survival (RFS) for a validated group in high-risk categories oscillated in individual models between 72 and 82\%, when only $9-39 \%$ of cases were assigned into high-risk groups.

Four other prognostic models were published more recently; all proposed models comprised a combination of several markers. ${ }^{5-18}$ In a group of 588 patients treated over 6 years, significant prognostic factors were stage, tumour grade, the ratio of positive/ removed LN and number of positive $L N .^{15}$ In a later study, a scoring system for LN-positive patients was suggested based on an analysis of 299 patients treated over 11 years, including tumour type, number of positive LN and tumour stage. ${ }^{16}$ Other authors used data obtained from the SEER database in 2004-2014 and tested a new marker, the log of odds between the number of removed pelvic $L N$ and the number of negative $L N .^{17}$ The result was a rather complicated nomogram that, in addition to markers listed above, also included age, race, marital status, tumour grade, FIGO stage, tumour type and tumour size. ${ }^{17}$ Recently, a Dutch group published an analysis of prognostic markers in a large multi-institutional database of 2124 cases in stages I/IIA treated within a 30 -year interval. ${ }^{18}$ Large tumour diameter, non-squamous tumour type, LN involvement, parametrial invasion, LVSI, deep stromal invasion and also less-radical surgery were identified as independent negative prognostic variables for survival. ${ }^{18}$

In our study, we compared the prognostic significance of 15 markers, which were assessed by imaging or by pathology according to a standardised protocol. None of the major principles of patient management, such as the selection of patients for primary surgical treatment, tailoring of surgical radicality, or criteria for adjuvant radiotherapy have changed during the study period. The management of patients in our series was unique in a few more aspects: (1) the majority of LN-negative patients did not receive adjuvant treatment; (2) SLN biopsy was routinely performed in the majority of the cohort; (3) SLNs were processed by an intensive pathological ultrastaging which increased the detection rate of MIC and small MAC.

Adenosquamous tumour type showed the highest $\mathrm{RR}$ in univariate analysis. Adenocarcinoma, on the contrary, was not associated with higher RR. In accordance with the majority of previous papers, LN involvement was a significant factor, both the presence of macrometastases and micrometastases. ${ }^{3-6,8,9,11}$ Parametrial invasion in our study was not significant for the prognosis. Our results cannot, however, be compared to other cohorts of patients which included patients with locally advanced stages. ${ }^{16-}$

${ }_{18}$ Only a limited number of cases with minimal invasion into parametria according to pre-operative imaging were selectively referred to primary surgery and enrolled in our study.

A separate analysis was conducted for patients with negative LN. These patients have an excellent prognosis, which makes most of the markers insignificant given the small number of recurrences. Even in this cohort, adenosquamous tumours and TFD were identified as individual significant prognostic markers. 


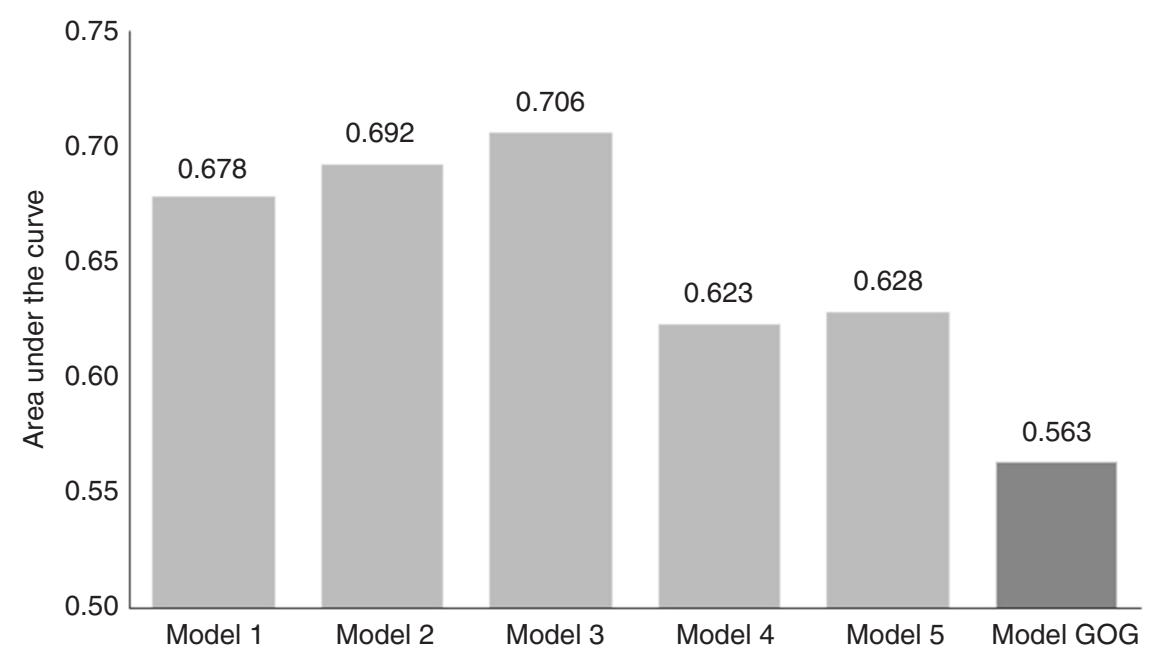

Fig. 5 Accuracy of prognostic models for disease recurrence in lymph node (LN)-negative patients (ROC analysis). Model 1: minimal tumour-free distance (TFD), tumour type; Model 2: TFD binarised, tumour type; Model 3: TFD binarised; tumour type, lymphovascular space invasion; Model 4: TFD binarised, tumour type, depth of stromal invasion; Model 5: largest tumour size binarised, tumour type; Model 6: GOG score.

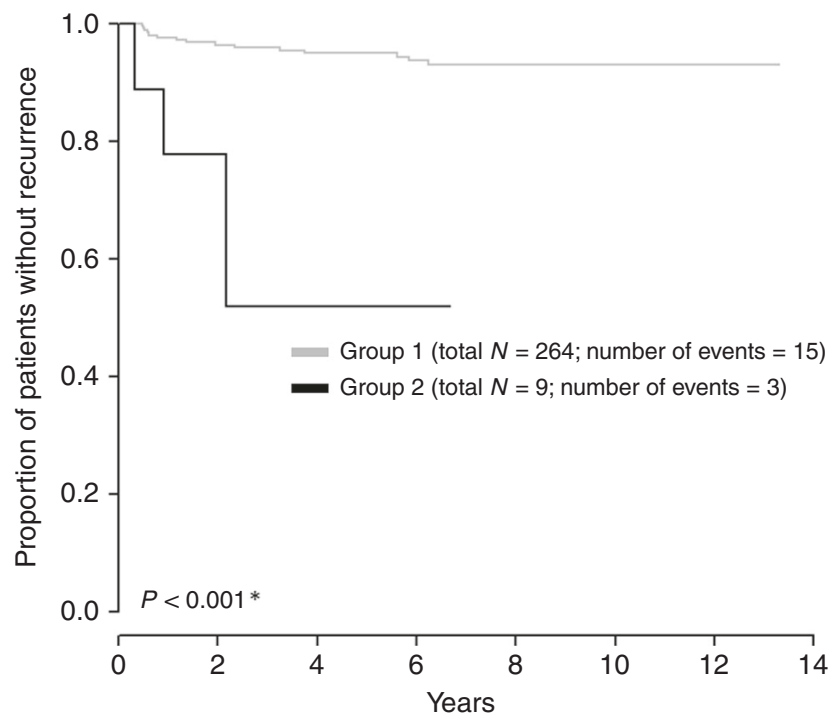

Fig. 6 Kaplan-Meier recurrence-free survival (RFS) curve for Model 2 in lymph node-negative patients. Group description (number of patients related to this characteristic is in the bracket): Group 1: other combinations not included in Group 2; Group 2: tumour-free distance $\leq 3.5^{* *}$, adenosquamous tumour type. One patient without event is not included into the groups: Minimal tupcf (US) $>3.5^{* *}$, adenosquamous tumour type. *Log-rank test. **Cutoff determined by ROC analysis, the criterion was the highest value of the sum of sensitivity and specificity.

All markers related to the assessment of tumour size were significant in univariate analysis and the most significant independent one was TFD. TFD also worked best in both prognostic models; the reliability of the models was substantially decreased if TFD was replaced by any other tumour-related markers. TFD is, however, not our invention. Several authors reported that the risk of LN involvement and poor survival is negatively associated with the thickness of the remaining fibromuscular cervical stroma around the tumour. Tsukamoto et al..$^{29}$ in 1966 and Noguchi et al. ${ }^{30}$ in 1983 reported a 5-year survival rate of $100 \%$ in patients in whom the remaining normal stroma measured more than $5 \mathrm{~mm}$ in thickness. In 1987, Kishi et al. ${ }^{31}$ reported a low risk of LN positivity (7\%) and high 5-year survival (92\%) in patients with uninvolved stroma $\geq 3 \mathrm{~mm}$, while corresponding figures were 37 and $26 \%$ if TFD was $\leq 3 \mathrm{~mm}$. Landoni et al..$^{32}$ in 1995 reported an increased risk of parametrial invasion in patients with TFD $\leq 3 \mathrm{~mm}$. In our study, TFD $(\leq 3.5 \mathrm{~mm})$ surpassed all other traditional markers which are related to tumour size. We can hypothesise that the distance between tumour and parametria better corresponds with the risk of extrauterine tumour spread than the tumour size or depth of stromal invasion, which does not take into account the size of the cervix and tumour location in the cervix. TFD in this study showed an inverse relationship to the presence of positive $L N$, thus supporting this hypothesis. TFD was by far the best marker in both predictive models for the entire population and for the LNnegative subgroup. TFD can be easily assessed by pre-operative imaging (MRI or expert US), and cut-off value was established at $3.5 \mathrm{~mm}$ so the factor could be binarised in predictive models.

The main limitation of the study is its retrospective design, which, however, can be partially compensated by the inclusion of all consecutive (eligible) patients. The main strength is the fact that all prognostic markers were assessed by a standardised protocol for imaging and pathology. Moreover, the majority of patients underwent SLN biopsy analysed by an intensive ultrastaging protocol, which increased the sensitivity of LN staging, especially detection of low volume disease (MIC and ITC). In addition, the treatment strategy in our institutional cohort was not significantly changed during the study period.

In conclusion, our study confirmed that LN involvement is a significant traditional prognostic factor for predicting RR in earlystage cervical cancer. Out of markers related to tumour size, we identified a new prognostic marker, TFD, which correlates the best with the recurrence risk and can be easily assessed by preoperative imaging. Prognostic models, combining TFD with LN status or tumour type in the entire population and in LN-negative patients, are easy to use in routine clinical practice and are able to identify the smallest possible group of patients with the highest risk of recurrence. Other traditional markers, such as LVSI or DSI, were less significant predictive factors and they did not improve prognostic models. Our models should be validated as indications for adjuvant treatment in future studies.

\section{AUTHOR CONTRIBUTIONS}

Conceptualisation: D.C. and L.D.; methodology: all authors; data analysis and statistics: J.J., S.S. and M.B.; investigation of patients: D.C., J.S., L.D., D.F., A.G., F.F., P.D., K.N., A.B. 
and R.K.; original draft preparation: D.C. and M.B.; review and editing: all authors. All authors have read and agreed to the published version of the paper.

\section{ADDITIONAL INFORMATION}

Ethics approval and consent to participate The study protocol was approved by the Institutional Review board of the leading institution (General University Hospital in Prague, Czech Republic) in 10/2017 under the registration number 1587/17 S-IV. Due to the retrospective nature of the study, a need for informed consent was waived by the Institutional Review board. The study was performed in accordance with the Declaration of Helsinki.

Consent to publish Not applicable.

Data availability The datasets used and/or analysed during the current study are available from the corresponding author on reasonable request.

Competing interests The authors declare no competing interests.

Funding information This work was supported by Charles University in Prague (UNCE 204065 and PROGRES Q28/LF1) and by a grant from the Czech Health Research Council (NV19-03-00023). The funders had no role in the design of the study; in the collection, analyses or interpretation of the data; in the writing of the paper or in the decision to publish the results.

Supplementary information is available for this paper at https://doi.org/10.1038/ s41416-020-01204-w.

Note This work is published under the standard license to publish agreement. After 12 months the work will become freely available and the license terms will switch to a Creative Commons Attribution 4.0 International (CC BY 4.0).

Publisher's note Springer Nature remains neutral with regard to jurisdictional claims in published maps and institutional affiliations.

\section{REFERENCES}

1. Cibula, D., Potter, R., Planchamp, F., Avall-Lundqvist, E., Fischerova, D., Haie Meder, C et al. The European Society of Gynaecological Oncology/European Society for Radiotherapy and Oncology/European Society of Pathology guidelines for the management of patients with cervical cancer. Radiother. Oncol. 127, 404-416 (2018).

2. Koh, W. J., Abu-Rustum, N. R., Bean, S., Bradley, K., Campos, S. M., Cho, K. R. et al. Cervical cancer, version 3.2019, NCCN clinical practice guidelines in oncology. J. Natl Compr. Canc Netw. 17, 64-84 (2019).

3. Delgado, G., Bundy, B., Zaino, R., Sevin, B.-U., Creasman, W. T. \& Major, F. Prospective surgical-pathological study of disease-free interval in patients with stage IB squamous cell carcinoma of the cervix: a Gynecologic Oncology Group study. Gynecol. Oncol. 38, 352-357 (1990).

4. Alvarez, R. D., Soong, S. J., Kinney, W. K., Reid, G. C., Schray, M. F., Podratz, K. C. et al. Identification of prognostic factors and risk groups in patients found to have nodal metastasis at the time of radical hysterectomy for early-stage squamous carcinoma of the cervix. Gynecol. Oncol. 35, 130-135 (1989).

5. Ho, C. M., Chien, T. Y., Huang, S. H., Wu, C. J., Shih, B. Y. \& Chang, S. C. Multivariate analysis of the prognostic factors and outcomes in early cervical cancer patients undergoing radical hysterectomy. Gynecol. Oncol. 93, 458-464 (2014).

6. Kamura, T., Tsukamoto, N., Tsuruchi, N., Saito, T., Matsuyama, T., Akazawa, K. et al. Multivariate analysis of the histopathologic prognostic factors of cervical cancer in patients undergoing radical hysterectomy. Cancer 69, 181-186 (1992).

7. Lai, C. H., Chang, C. J., Huang, H. J., Hsueh, S., Chao, A., Yang, J. E. et al. Role of human papillomavirus genotype in prognosis of early-stage cervical cancer undergoing primary surgery. J. Clin. Oncol. 25, 3628-3634 (2007).

8. Sevin, B. U., Lu, Y., Bloch, D. A., Nadji, M., Koechli, O. R. \& Averette, H. E. Surgically defined prognostic parameters in patients with early cervical carcinoma. A multivariate survival tree analysis. Cancer 78, 1438-1446 (1996).

9. Yuan, C. C., Wang, P. H., Lai, C. R., Yen, M. S., Chen, C. Y. \& Juang, C. M. Prognosispredicting system based on factors related to survival of cervical carcinoma. Int. J. Gynaecol. Obstet. 63, 163-167 (1998).

10. Kristensen, G. B., Abeler, V. M., Risberg, B., Trop, C. \& Bryne, M. Tumor size, depth of invasion, and grading of the invasive tumor front are the main prognostic factors in early squamous cell cervical carcinoma. Gynecol. Oncol. 74, 245-251 (1999).
11. Grisaru, D. A., Covens, A., Franssen, E., Chapman, W., Shaw, P., Colgan, T. et al. Histopathologic score predicts recurrence free survival after radical surgery in patients with stage IA2-IB1-2 cervical carcinoma. Cancer 97, 1904-1908 (2003).

12. Sedlis, A., Bundy, B. N., Rotman, M. Z., Lentz, S. S., Muderspach, L. I. \& Zaino, R. J. A randomized trial of pelvic radiation therapy versus no further therapy in selected patients with stage IB carcinoma of the cervix after radical hysterectomy and pelvic lymphadenectomy: a Gynecologic Oncology Group Study. Gynecol. Oncol. 73, 177-183 (1999)

13. Van de Putte, G., Lie, A. K., Vach, W., Baekelandt, M. \& Kristensen, G. B. Risk grouping in stage IB squamous cell cervical carcinoma. Gynecol. Oncol. 99, 106-112 (2005).

14. Sartori, E., Tisi, G., Chiudinelli, F., La Face, B., Franzini, R. \& Pecorelli, S. Early stage cervical cancer: adjuvant treatment in negative lymph node cases. Gynecol. Oncol. 107, 170-174 (2007).

15. Chen, Y., Zhang, L., Tian, J., Fu, X., Ren, X. \& Hao, Q. Significance of the absolute number and ratio of metastatic lymph nodes in predicting postoperative survival for the International Federation of Gynecology and Obstetrics stage IA2 to IIA cervical cancer. Int. J. Gynecol. Cancer 23, 157-163 (2003).

16. Lee, Y. J., Kim, D. Y., Lee, S. W., Park, J. Y., Suh, D. S., Kim, J. H. et al. A postoperative scoring system for distant recurrence in node-positive cervical cancer patients after radical hysterectomy and pelvic lymph node dissection with para-aortic lymph node sampling or dissection. Gynecol. Oncol. 144, 536-540 (2007).

17. Wang, C., Yang, C., Wang, W., Xia, B., Li, K., Sun, F. et al. A prognostic nomogram for cervical cancer after surgery from SEER database. J. Cancer 9, 3923-3928 (2018).

18. Derks, M., van der Velden, J., de Kroon, C. D., Nijman, H. W., van Lonkhuijzen, L, van der Zee, A. G. J. et al. Surgical treatment of early-stage cervical cancer: a multi-institution experience in 2124 cases in The Netherlands over a 30-year period. Int. J. Gynecol. Cancer 28, 757-763 (2018).

19. Biewenga, P., van der Velden, J., Mol, B. W., Stalpers, L. J., Schilthuis, M. S., van der Steeg, J. W. et al. Validation of existing prognostic models in patients with earlystage cervical cancer. Gynecol. Oncol. 115, 277-284 (2009).

20. Cibula, D. \& Abu-Rustum, N. R. Pelvic lymphadenectomy in cervical cancersurgical anatomy and proposal for a new classification system. Gynecol. Oncol. 116, 33-37 (2010).

21. Querleu, D., Cibula, D. \& Abu-Rustum, N. R. 2017 update on the Querleu-Morrow classification of radical hysterectomy. Ann. Surg. Oncol. 24, 3406-3412 (2017).

22. Testa, A. C., Ludovisi, M., Manfredi, R., Zannoni, G., Gui, B., Basso, D. et al. Trans vaginal ultrasonography and magnetic resonance imaging for assessment of presence, size and extent of invasive cervical cancer. Ultrasound Obstet. Gynecol. 34, 335-344 (2009).

23. Fischerova, D., Cibula, D., Stenhova, H., Vondrichova, H., Calda, P., Zikan, M. et al Transrectal ultrasound and magnetic resonance imaging in staging of early cervical cancer. Int. J. Gynecol. Cancer 18, 766-772 (2008).

24. Epstein, E., Testa, A., Gaurilcikas, A., Di Legge, A., Ameye, L., Atstupenaite, V. et al Early-stage cervical cancer: tumor delineation by magnetic resonance imaging and ultrasound-a European multicenter trial. Gynecol. Oncol. 128, 449-453 (2013).

25. Chiappa, V., Di Legge, A., Valentini, A. L., Gui, B., Micco, M., Ludovisi, M. et al. Agreement of two-dimensional and three-dimensional transvaginal ultrasound with magnetic resonance imaging in assessment of parametrial infiltration in cervical cancer. Ultrasound Obstet. Gynecol. 45, 459-469 (2015).

26. Education and Practical Standards Committee, European Federation of Societies for Ultrasound in Medicine and Biology. Minimum training recommendations for the practice of medical ultrasound. Ultraschall Med. 27, 79-105 (2006).

27. Fischerova, D. Ultrasound scanning of the pelvis and abdomen for staging of gynecological tumors: a review. Ultrasound Obstet. Gynecol. 38, 246-266 (2011).

28. Cibula, D. \& McCluggage, W. G. Sentinel lymph node (SLN) concept in cervical cancer: Current limitations and unanswered questions. Gynecol. Oncol. 152, 202-207 (2019).

29. Tsukamoto, T. The studies on the spreading modus of the uterine cervical cancer: especially a new proposal of the postoperative histological classification. Shinshu Med J. 15, 354-383 (1966).

30. Noguchi, H., Shiozawa, K., Tsukamoto, T., Tsukahara, Y., Iwai, S. \& Fukuta, T. The postoperative classification for uterine cervical cancer and its clinical evaluation. Gynecol. Oncol. 16, 219-231 (1983).

31. Kishi, Y., Hashimoto, Y., Sakamoto, Y. \& Inui, S. Thickness of uninvolved fibromuscular stroma and extrauterine spread of carcinoma of the uterine cervix. Cancer 60, 2331-2336 (1987).

32. Landoni, F., Bocciolone, L., Perego, P., Maneo, A., Bratina, G. \& Mangioni, C. Cancer of the cervix, FIGO stages IB and IIA: patterns of local growth and paracervical extension. Int. J. Gynecol. Cancer 5, 329-334 (1995). 